\title{
Tratamiento de las fracturas femorales periprotésicas Vancouver B3 con injerto óseo impactado. Supervivencia y complicaciones
}

\author{
Fernando Díaz Dilernia," José I. Oñativia," Pablo Slullitel," Martín Buttaro" \\ "Centro de Cadera "Sir John Charnley", Instituto de Ortopedia y Traumatología “Carlos E. Ottolenghi”, Hospital Italiano de Buenos Aires, \\ Ciudad Autónoma de Buenos Aires, Argentina \\ ***Traumatología del Norte, Tucumán, Argentina
}

\section{RESUMEN}

Objetivos: El tratamiento de elección para las fracturas femorales periprotésicas Vancouver B3 aún no está definido. Por este motivo, nos propusimos analizar la tasa de complicaciones de la técnica de injerto óseo impactado con un vástago cementado cuando se utiliza para tratar estas fracturas. Materiales y Métodos: Estudiamos retrospectivamente 33 fracturas femorales periprotésicas B3 tratadas con la técnica de injerto óseo impactado operados entre 2000 y 2016, analizando la tasa de complicaciones. La mediana de seguimiento fue de 75 meses (RIC 36-111). La mediana de edad fue de 78 años (RIC 74-83). La mediana del defecto óseo femoral fue 3 (RIC 3-3) según la clasificación de la Endo-Klinik. Se realizó un análisis de regresión múltiple para determinar los factores de riesgo asociados a complicaciones, las variables incluidas fueron: cantidad de cirugías previas, diámetro de la nueva cabeza femoral y defecto óseo femoral. Resultados: Se realizó una cirugía de revisión en dos etapas en cuatro pacientes. Se registraron cinco fallas asépticas del implante y dos luxaciones en toda la serie. El análisis de regresión lineal multivariable mostró una asociación significativa entre el grado del defecto óseo femoral Endo-Klinik y la tasa de complicaciones $(p=0,04)$. Conclusión: La reconstrucción femoral con la técnica de injerto óseo impactado para tratar fracturas periprotésicas Vancouver B3 provocó una alta tasa de complicaciones.

Palabras clave: Fractura femoral periprotésica B3; técnica de injerto óseo impactado; complicaciones; análisis de supervivencia. Nivel de Evidencia: IV

\section{Impaction Bone Grafting for the Treatment of Vancouver Type B3 Periprosthetic Femoral Fractures.} Survivorship and Complication Rate Analysis

\section{ABSTRACT}

Aims: Because the gold standard for the treatment of Vancouver type B3 periprosthetic femoral fractures (PFFs) is yet to be defined, we sought to analyze the complication rate of the impaction bone grafting (IBG) technique with a cemented stem for the treatment of this fractures. Materials and Methods: We retrospectively studied 33 B3 PFFs treated with the IBG technique operated between 2000 and 2016, analyzing the complication rate. The median follow-up was 75 months (interquartile range [IQR], 36-111). The median age was 78 years (IQR, 74-83). The median grade of EndoKlinik femoral bone defect was 3 (IQR, 3-3). We performed a multiple regression analysis to determine risk factors for complications, including the following variables: number of previous surgeries, femoral head diameter, and femoral bone defect. Results: As for infection outcomes, 2-stage revision surgery was performed in 4 patients. We registered 5 implant failures and 2 dislocations in the whole series. Multiple regression analysis showed a significant association between the grade of EndoKlinik femoral bone defect and complication rate $(P=0.04)$. Conclusion: Femoral reconstruction with the IBG technique evidenced a high complication rate for the treatment of B3 PFF. Key words: B3 periprosthetic femoral fractures; impaction bone grafting technique; complications; survivorship analysis. Level of Evidence: IV 


\section{INTRODUCCIÓN}

Una de las complicaciones más complejas después de la artroplastia total de cadera es la fractura femoral periprotésica (FFP). Varios estudios informaron una prevalencia del 1,7\% y 6,2\% a los 10 años de seguimiento después de la artroplastia total de cadera primaria y de revisión, respectivamente. ${ }^{1,2}$ Sin embargo, debido al esperado aumento anual de las artroplastias totales de cadera primarias y de revisión, es probable que haya un aumento exponencial de las FFP en las próximas décadas. ${ }^{3}$ Para lograr un resultado exitoso en estos casos, se requiere de un cirujano entrenado en trauma y reconstrucción articular, dada la complejidad de la enfermedad y la exigencia de la técnica quirúrgica. ${ }^{1-4}$

Las FFP alrededor de un vástago fijo (Vancouver tipo B1) se tratan con fijación interna, y las fracturas alrededor de un implante flojo con una calidad ósea adecuada (Vancouver tipo B2) se tratan con la revisión de la artroplastia total de cadera. Una FFP sobre un vástago flojo con pérdida de capital óseo (Vancouver tipo B3) plantea el desafío de lograr simultáneamente la estabilidad del implante y la consolidación de la fractura. En este sentido, la elección del implante para tratar la fractura y reconstruir el fémur es de vital importancia. ${ }^{5}$ Recientemente, el Registro Nacional Sueco de Artroplastia de Cadera informó una prevalencia de FFP B3 del 4,1\% (43 de 1049) en un período de 21 años. ${ }^{6}$

Las FFP B3 representan un desafío incluso para el cirujano con más experiencia. Se han descrito varias técnicas quirúrgicas para su tratamiento: ${ }^{7}$ el injerto óseo impactado (IOI), el aloinjerto óseo de banco, la fijación distal modular e incluso el reemplazo femoral proximal. La técnica con IOI fue una de las opciones de reconstrucción más utilizadas hasta el advenimiento de la fijación distal no cementada, ${ }^{1}$ teniendo en cuenta que esta última es una técnica menos exigente y no requiere de un banco de huesos. Por otro lado, la fijación distal agregó la opción de modularidad, aumentando así la versatilidad para reconstruir la biomecánica del fémur proximal. ${ }^{8,9}$ Aunque los vástagos modulares cónicos de fijación distal han logrado una supervivencia del $90 \%$ y buenos resultados funcionales en el mediano plazo, ${ }^{10,11}$ existen algunos patrones de fractura con pérdida ósea masiva y una geometría del canal femoral desfavorable, como un fémur proximal tipo $\mathrm{C}$ de la clasificación de Dorr, que dificultan su indicación. ${ }^{11}$

La evidencia relacionada con el tratamiento de las FFP B3 se compone principalmente de series de casos pequeñas con un seguimiento a corto plazo. ${ }^{12-15}$ En este escenario, no hay acuerdo en el tratamiento óptimo para estos pacientes. Por lo tanto, el objetivo de nuestro estudio fue analizar la tasa de complicaciones asociada a la técnica de IOI para tratar la FFP Vancouver B3.

\section{MATERIALES Y MÉTODOS}

Después de obtener la aprobación del Comité de Ética en Investigación de la institución, estudiamos retrospectivamente una serie de 35 pacientes con FFP y aflojamiento del tallo y falta de capital óseo en el fémur proximal, tratados con la técnica de IOI entre 2000 y 2016. Todas las fracturas eran Vancouver tipo B3, ya que estaban ubicadas alrededor de un componente femoral flojo y se asociaban con un capital óseo deficiente en el fémur proximal. ${ }^{6}$ Durante el período de estudio, también se trataron 43 FFP tipo A, 193 tipo B1 y 99 tipo B2 que no se incluyeron en el estudio. Se excluyeron dos casos de la serie por el antecedente de infección periprotésica antes de la FFP, por lo que se analizaron 33 casos. En el momento de la revisión final, nueve pacientes habían fallecido y no se había perdido ningún otro paciente durante el seguimiento. La mediana de seguimiento de estos nueve pacientes fue de 58 meses (rango intercuartílico [RIC] 35-99). Toda la información se obtuvo de la base de datos electrónica recopilada prospectivamente de la institución, digitalizada en 2004. Solo seis pacientes habían sido operados antes en nuestra institución. Se clasificó el capital óseo con radiografías anteroposterior y lateral del fémur afectado antes de la cirugía, confirmando estos hallazgos luego de la extracción de los componentes, de acuerdo con la clasificación de la Endo-Klinik. ${ }^{16}$ Las características demográficas de la serie se detallan en la Tabla 1.

Dentro del período de estudio, el mismo grupo experimentado de cirujanos de cadera realizó todas las cirugías en quirófanos con flujo laminar siguiendo los principios generales que se han descrito para la técnica original (Tabla 2). ${ }^{4}$ Bajo anestesia epidural hipotensiva, se realizó un abordaje posterolateral extendido en 29 pacientes y un abordaje transtrocantérico en los cuatro restantes. Se descartó sistemáticamente una infección concomitante enviando muestras intraoperatorias para el estudio anatomopatológico. ${ }^{17}$ Todos los tallos extraídos fueron cementados. El componente femoral, el manto de cemento y los residuos de polimetilmetacrilato, así como la interfaz circundante, se eliminaron completamente para identificar la cantidad de hueso femoral remanente. El componente acetabular se revisó en 25 casos para adaptarse al tamaño de la nueva cabeza femoral implantada. Los tallos extraídos se detallan en la Tabla 1. 
Tabla 1. Características demográficas de la serie

\begin{tabular}{|l|c|}
\hline Variable & Serie (n = 33) \\
\hline Mediana de la edad (años) & 78 (RIC 74-83) \\
\hline Sexo (femenino/masculino) & $23 / 10$ \\
\hline Mediana de cirugías previas (n) & 1 (RIC 1-2) \\
\hline Mediana de la clasificación de la Endo-Klinik & 3 (RIC 3-3) \\
\hline Diagnóstico inicial (n) & \\
\hline Osteoartritis primaria & 20 \\
\hline Displasia de cadera & 6 \\
\hline Fractura de cuello femoral & 7 \\
\hline Tallo extraído (n) & \\
Charnley & 12 \\
Charnley Elite-Plus & 10 \\
\hline C-STEM@ & 5 \\
\hline Müller & 3 \\
\hline Harris-Galante & 1 \\
\hline Exeter & 2 \\
CPT & 0 \\
\hline Mediana de seguimiento (meses) & 75 (RIC 36-111) \\
\hline RIC = rango intercuartílico. & \\
\hline
\end{tabular}

Tabla 2. Técnica de reconstrucción utilizada

\begin{tabular}{|l|c|}
\hline Variable & Serie (n = 33) \\
\hline Mediana de tablas de injerto cortical (n) & 0 (RIC 0-1) \\
\hline Mediana de injertos de cabezas femorales (n) & 2 (RIC 2-3) \\
\hline Mediana de mallas metálicas (n) & 1 (RIC 0-1) \\
\hline Mediana de cerclaje de alambres (n) & 6 (RIC 5-8) \\
\hline Tallo femoral implantado (n) & \\
\hline Charnley & 1 \\
\hline Exeter & 12 \\
\hline C-STEM® & 20 \\
\hline Mediana del diámetro de la cabeza femoral (mm) & 28 (RIC 28-28) \\
\hline Revisión acetabular & 25 \\
\hline Sí & 8 \\
\hline No & \\
\hline RIC $=$ rango intercuartílico.
\end{tabular}

Se obtuvieron aloinjertos óseos congelados del banco de huesos de la institución siguiendo el protocolo de la American Association of Tissue Banks para la recolección y el procesamiento de injertos. ${ }^{18}$ Los injertos se impactaron de acuerdo con la técnica descrita por Gie y cols., ${ }^{19}$ utilizando instrumentos de impacto primario (DePuy International, Leeds, Reino Unido) y el sistema de instrumentos para cirugías de revisión (Stryker, Newbury, Reino Unido). Se pretendía obtener un espesor de hueso esponjoso de $10 \mathrm{~mm}$ en la parte proximal del fémur reconstruido. Se utilizaron cerclajes de alambres y mallas metálicas cuando fue necesario. Los detalles de la técnica de reconstrucción se describen en la Tabla 2. 
La reconstrucción femoral se realizó en todos los casos con tallos largos cementados (Tabla 2 y Figura 1). Inyectamos, de manera retrógrada, cemento quirúrgico CMW con gentamicina (DePuy, Leeds, Reino Unido) en 21 casos y cemento Simplex con tobramicina (Stryker, Rutherford, New Jersey, EE.UU.) en los 12 restantes.

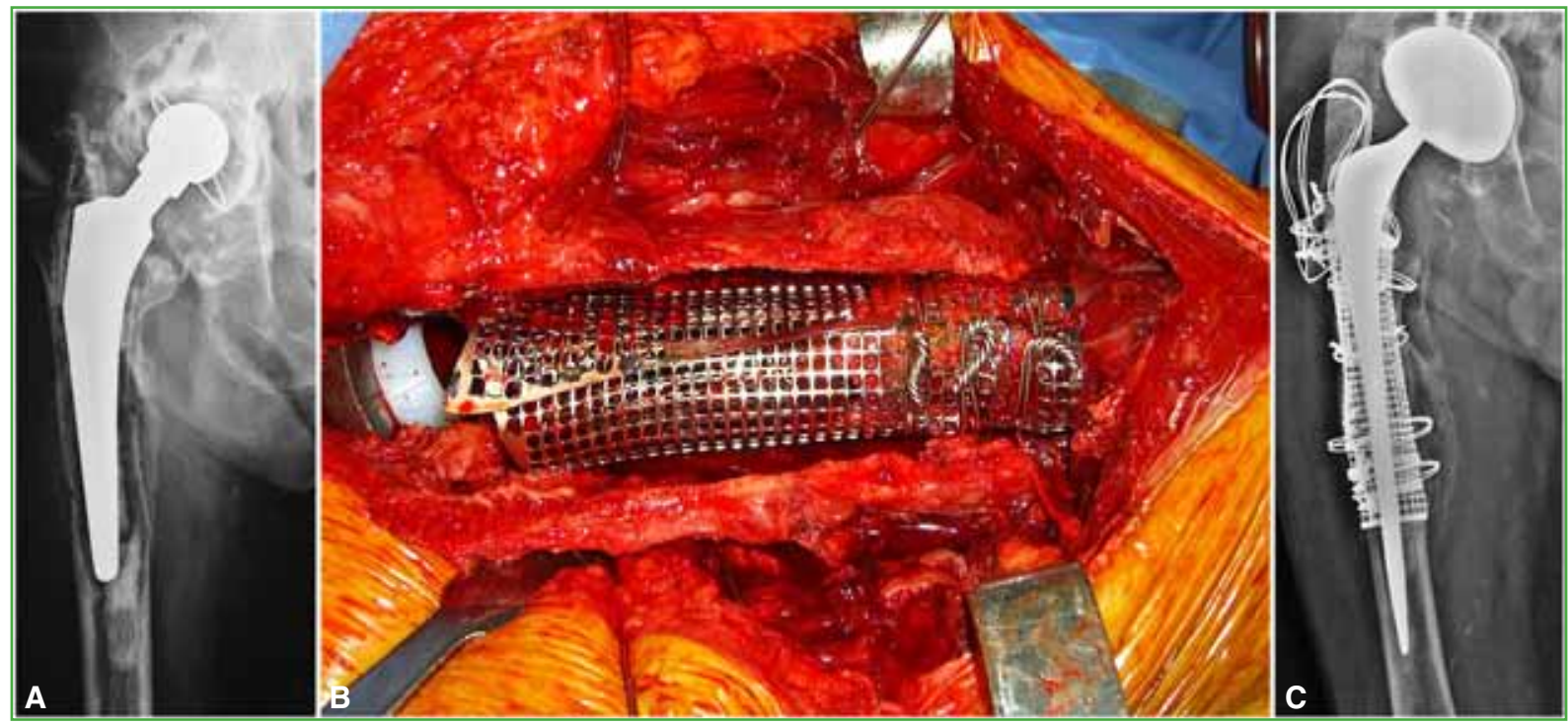

Figura 1. Técnica de injerto óseo impactado. A. Radiografía anteroposterior de cadera derecha preoperatoria que muestra una fractura femoral periprotésica. B. Imagen intraoperatoria. Se observan el injerto óseo impactado, las mallas metálicas y el cerclaje de alambres. C. Radiografía anteroposterior de cadera derecha después de la reconstrucción femoral con un tallo largo cementado.

Se administró profilaxis de rutina para la enfermedad tromboembólica con enoxaparina $40 \mathrm{mg} /$ día, por vía subcutánea, durante el primer mes posoperatorio. ${ }^{20}$ No prescribimos profilaxis de rutina contra la calcificación heterotópica. El protocolo de rehabilitación incluyó la movilización temprana 48 h después de la cirugía, la deambulación con un andador y el contacto con el pie sobre el lado operado durante 90 días. Después se indicó progresivamente la carga de peso, según la tolerancia, con el uso de un bastón durante, al menos, un mes, según la incorporación del injerto y la consolidación de la osteotomía, y la fractura observadas en las radiografías de control.

Las radiografías anteroposterior y lateral de la pelvis y el fémur operados se tomaron inmediatamente después de la cirugía, a los 45 días, a los 3, 6 y 12 meses, y luego una vez al año. A los fines de este estudio, se estableció contacto con todos los pacientes para una cita de seguimiento radiográfico. Se consideró consolidación de la fractura/osteotomía cuando había evidencia radiográfica de puente óseo en ambas proyecciones o ausencia del trazo fracturario. ${ }^{21,22} \mathrm{Se}$ compararon las radiografías tomadas inmediatamente después de la operación con las del último control. La radiolucencia femoral, definida como cualquier línea irregular entre el vástago y la interfaz ósea; y la osteólisis periprotésica, definida como pérdida ósea progresiva $>5 \mathrm{~mm}$, se evaluaron mediante las zonas de Gruen..$^{23}$ La subsidencia del vástago femoral se determinó mediante el método descrito por Loudon y Charnley, ${ }^{24}$ que mide la distancia desde un punto seleccionado (pero variable) en la prótesis femoral hasta un punto fijo en el hueso. El aflojamiento se definió como un hundimiento $>5 \mathrm{~mm}$ o una demarcación progresiva alrededor del vástago. La fijación del tallo se evaluó utilizando los criterios de Engh. ${ }^{25}$ Se consideró una falla séptica a cualquier caso que requiriera una cirugía de revisión debido a una infección en el sitio quirúrgico. Se consideró una falla aséptica del implante cuando se hallaron signos de aflojamiento, fractura del vástago o una nueva fractura periprotésica, que requirieron una cirugía de revisión.

Las variables continuas se expresan como medianas y RIC por tener una distribución no gaussiana. Las variables categóricas se expresan como frecuencias y porcentajes. Las variables continuas se compararon utilizando la prueba U de Mann-Whitney, dado que los datos tenían una distribución anormal. Las variables categóricas se compararon utilizando las pruebas de ji al cuadrado y exacta de Fisher. El análisis de supervivencia según Kaplan-Meier 
se estimó considerando como punto final de seguimiento a la cirugía de revisión sin importar la causa. Se realizó un análisis de regresión lineal multivariable para determinar los factores de riesgo independientes asociados a las complicaciones. Se incluyeron las variables que se consideraron significativas en el análisis univariado o con relevancia clínica. Los coeficientes de regresión (CR) se informan con sus intervalos de confianza del 95\% (IC95\%). Se consideró estadísticamente significativo un valor $\mathrm{p}<0,05$. Se empleó el programa estadístico Stata $13^{\mathrm{TM}}$ (Stata Corp., College Station, Texas, EE.UU.) para el análisis de los datos.

\section{RESULTADOS}

Se produjeron 11 complicaciones quirúrgicas en la serie. En cuanto a las infecciones periprotésicas, cuatro (12\%) pacientes desarrollaron una infección profunda en una mediana de 17 meses (RIC 14-20) y fueron tratados con una cirugía de revisión en dos tiempos, sin recurrencias en una mediana de seguimiento de 22 meses (RIC 18-28) después del reimplante.

Se registraron cinco (15\%) fallas asépticas del implante: un aflojamiento femoral de un tallo de Charnley a los 47 meses de seguimiento tratado con una cirugía de revisión con una nueva técnica de IOI; una fractura femoral atraumática Vancouver B1 tratada con reducción abierta y fijación interna, y tres fracturas atraumáticas del vástago femoral, todas tallos C-STEM largos (DePuy International, Leeds, Reino Unido) (Figura 2). Estas últimas tres ocurrieron en una mediana de 60 meses (RIC 44-86) desde la cirugía inicial y se revisaron de inmediato con un vástago modular no cementado ( 2 casos) y una nueva técnica de IOI en el caso restante.
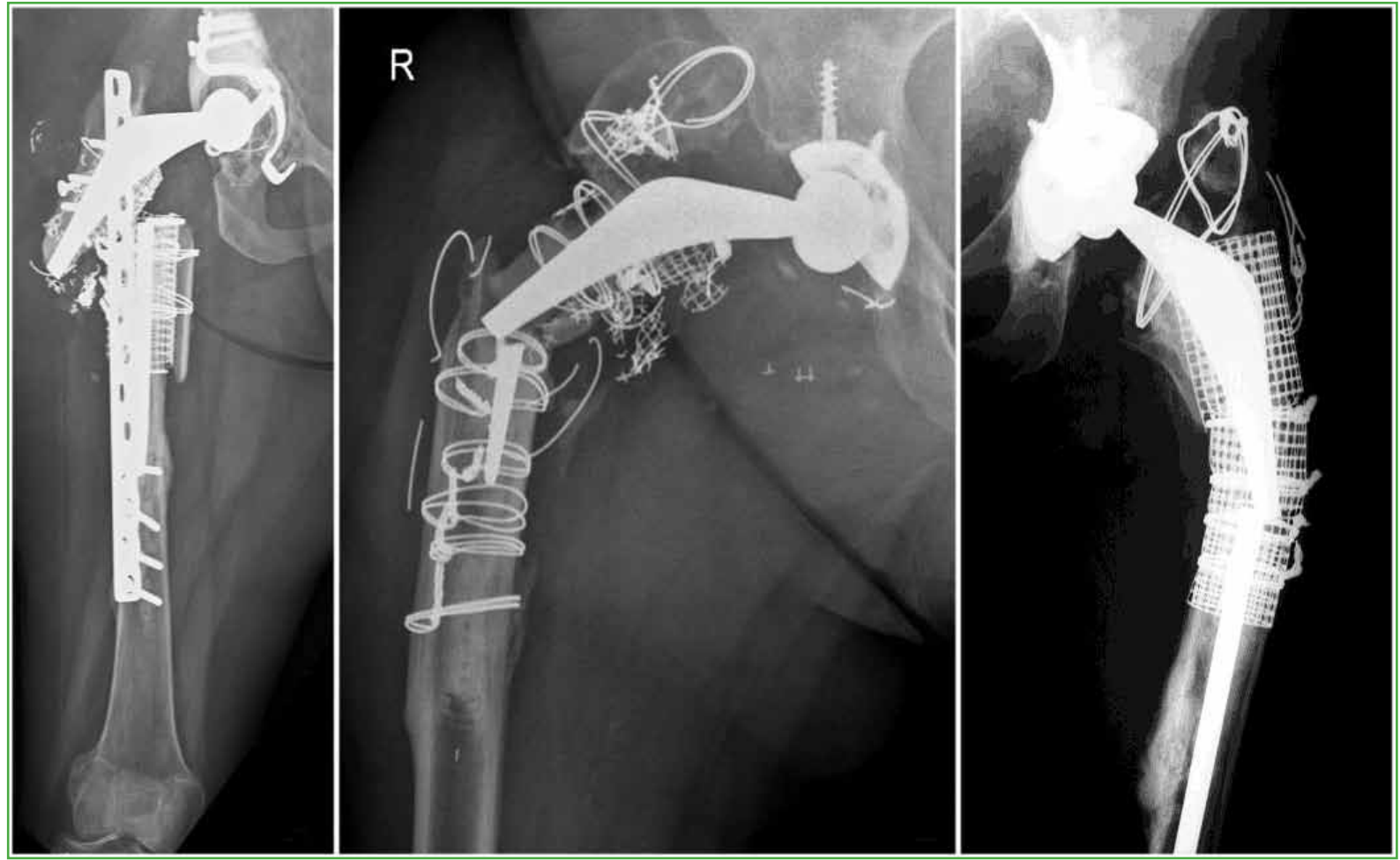

Figura 2. Radiografías anteroposteriores de fémur y cadera preoperatorias que muestran los tres tallos C-STEM fracturados.

Dos $(6 \%)$ pacientes tuvieron episodios de inestabilidad recurrente. Todos ocurrieron en casos operados con una cabeza femoral de $28 \mathrm{~mm}$ de diámetro. Un paciente presentó dos episodios de inestabilidad tratados, en forma conservadora, con reducción cerrada y una férula en abducción de cadera por seis semanas. Aunque se indicó la cirugía de revisión, el paciente prefirió el tratamiento conservador. El otro paciente sufrió una inestabilidad recurrente y, por lo tanto, se revisó con un liner constreñido y cementado a los 10 meses de la cirugía. No se detectaron nuevos episodios de inestabilidad en estos pacientes al final del seguimiento. 
Uno de los cuatro pacientes operados por un abordaje transtrocantérico desarrolló una seudoartrosis asintomática del trocánter mayor que fue tratada en forma conservadora. Hubo seis casos de hundimiento de hasta 2 mm; todos los tallos se consideraron estables con signos de incorporación ósea según los criterios de Engh ${ }^{25}$ al final del seguimiento. No hubo casos de osteólisis periprotésica.

Se registraron también otras complicaciones médicas menores que no se incluyeron en el análisis de supervivencia. Cuatro pacientes tuvieron trombosis venosa profunda y otros dos, una neumonía durante su estancia hospitalaria.

El análisis de regresión lineal multivariable mostró una asociación significativa entre el grado del defecto óseo femoral y el desarrollo de complicaciones (CR -0,45; IC95\% de -0,91 a 0,01; p =0,04) (Tabla 3). Con la revisión del vástago femoral como punto final, la supervivencia del implante fue del $73 \%$ en una mediana de 75 meses (RIC 36-111) (Figura 3).

Tabla 3. Complicaciones registradas en la serie

\begin{tabular}{|l|c|}
\hline Complicación & Serie $(\mathbf{n}=\mathbf{3 3})$ \\
\hline Infección, n (\%) & $4(12 \%)$ \\
\hline Falla aséptica del implante, n (\%) & $5(15 \%)$ \\
\hline Luxación, n (\%) & $2(6 \%)$ \\
\hline
\end{tabular}

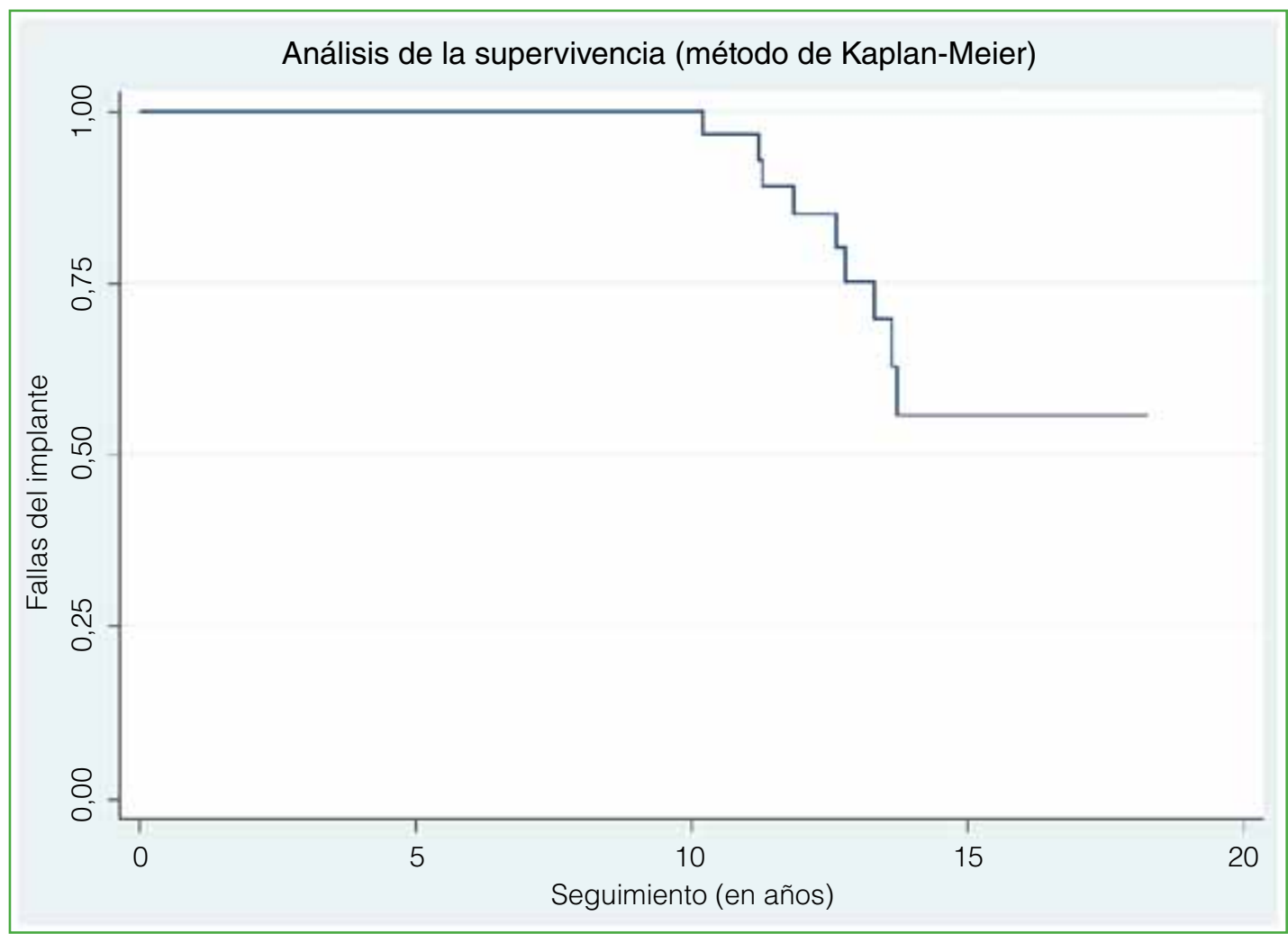

Figura 3. Análisis de la supervivencia de la técnica de injerto óseo impactado utilizando el método de KaplanMeier. Con la revisión del vástago femoral como punto final, la supervivencia del implante fue del 73\% en una mediana de 75 meses (RIC 36-111). 


\section{DISCUSIÓN}

En esta serie retrospectiva, la técnica de IOI tuvo una alta tasa de complicaciones posoperatorias. El tratamiento ideal de las FFP Vancouver B3 continúa siendo controvertido. Se reportaron varias opciones terapéuticas, como el IOI, la fijación distal modular, la aloprótesis e incluso el reemplazo femoral proximal. ${ }^{26}$ Todas han logrado buenos resultados a corto plazo. No obstante, todavía no existe un algoritmo razonable para elegir una técnica por sobre la otra.

Nuestro estudio no estuvo exento de limitaciones. Primero, por su naturaleza retrospectiva, se vio afectado por los sesgos inherentes a este diseño metodológico. El tamaño de la muestra dio lugar a un pequeño número de casos incluidos, lo que restringió la producción de un análisis estadístico más preciso; sin embargo, se trata de un subgrupo de pacientes altamente seleccionados en una enfermedad de baja prevalencia. De esta manera, se puede argumentar que la falta de significación estadística observada para algunas de las variables analizadas puede ser el resultado de un error de tipo beta (estudio de bajo poder). Sin embargo, es uno de los estudios más grandes que analizan los resultados quirúrgicos de esta técnica en casos de FFP B3. Segundo, no hemos analizado los resultados clínicofuncionales de este método de reconstrucción. En este sentido, el dolor posoperatorio, la marcha anormal y la capacidad para realizar las actividades de la vida diaria podrían haber agregado más información sobre las expectativas fallidas de los pacientes, lo que puede ser subestimado únicamente analizando el advenimiento de una nueva cirugía de revisión. En tercer lugar, creemos que nuestra tasa de supervivencia debe considerarse como la mejor estimación de casos. Dado que hubo un período de seguimiento amplio y heterogéneo, esperamos que algunos de estos pacientes aún se sometan a una artroplastia de revisión por cualquier motivo, con un seguimiento más prolongado. En cuarto lugar, nuestros datos no incluían información completa sobre los datos demográficos y las comorbilidades específicas o los factores perioperatorios que contribuyeran a una infección o una luxación, ya que fueron las complicaciones más comunes. Por lo tanto, nuestros resultados de complicaciones también deben considerarse como las mejores estimaciones de casos. En quinto lugar, debido a la mala calidad de las radiografías analógicas y escaneadas en nuestra base de datos de aquellos pacientes operados antes de 2003, no se pudo utilizar la clasificación de Barrack. Como muchos de los defectos fueron reconstruidos con mallas metálicas, esta fue una limitante agregada a la hora de realizar una evaluación precisa de la técnica de cementación y de la incorporación ósea.

Aunque los vástagos femorales largos, totalmente porosos y no cementados se pueden usar de manera eficaz para tratar la mayoría de estas fracturas ${ }^{11}$ hay un grupo de pacientes con patrones fracturarios específicos, pérdida ósea masiva por debajo del istmo femoral y una geometría desfavorable del canal femoral que excluyen el uso de estos implantes. En 2004, Tsiridis y cols. ${ }^{27}$ informaron 106 casos con FFP Vancouver tipo B2 y B3, a 89 se los sometió a una revisión cementada con IOI y, a los 17 restantes, a una revisión cementada sin IOI. Los autores hallaron que los pacientes tratados con IOI y un vástago largo que sobrepasaban el trazo distal de fractura tenían significativamente más probabilidades de consolidación que los que tenían un vástago largo sin IOI. Más recientemente, Li y cols. ${ }^{28}$ evaluaron retrospectivamente el resultado de 33 FFP Vancouver B3, 17 tratadas con IOI y 16, con IOI combinado con aloinjerto estructural cortical. Los autores comunicaron una tasa de reoperación del 9\%, con un tallo estable con incorporación ósea en 24 pacientes, con una fijación fibrosa en ocho y sin fijación alguna en el paciente restante. Aunque este procedimiento es técnicamente demandante, se ha demostrado que restaura el capital óseo con una tasa de supervivencia del 87,7\% a los 20 años de seguimiento tomando como punto final la revisión por cualquier motivo. ${ }^{29}$ Sin embargo y como describimos en este estudio, el IOI no está exento de complicaciones. Se produjeron cinco fallas asépticas del implante, incluidas tres fracturas del vástago femoral atraumáticas. Una revisión retrospectiva del Registro Nacional Sueco ${ }^{30}$ reveló 1305 casos de IOI con una supervivencia del 94\% a los 15 años, incluyendo todas las causas de fracaso. Curiosamente, no hubo diferencias en la supervivencia entre un componente femoral largo y otro más corto. La mayoría de las complicaciones que requirieron una revisión ocurrieron dentro de los cuatro años y consistieron en infección y fractura femoral (33 casos, 47,5\%), aflojamiento aséptico (11 casos, 15,7\%) y hundimiento (13 casos, 18,6\%). Las fracturas por fatiga del implante también pueden ocurrir cuando el soporte óseo proximal es inapropiado, ${ }^{31} \mathrm{y}$ es posible evitarlas si se siguen los estrictos principios de la técnica original, que se ha refinado mediante el desarrollo de un sistema de revisión femoral instrumentado. ${ }^{32}$ Se comunicó que un índice de masa corporal $>30$, el uso de aloinjerto óseo irradiado y un grado de Endo-Klinik avanzado son factores predictivos independientes de fracaso. ${ }^{33,34}$ Aunque no pudimos analizar el índice de masa corporal como una variable independiente, todos los casos incluidos tenían un gran defecto óseo proximal, lo que podría haber estado implícito en la génesis de las fracturas de implantes descritas. ${ }^{31}$ Aunque es especulativo, el vástago largo cementado mostró cierta intolerancia a la pérdida de integridad del fémur proximal, que resultó en tres fracturas del implante. 
La revisión con un vástago modular no cementado es otra opción factible, siempre que haya suficiente hueso para la fijación distal y no haya una conminución que se extienda hacia la metáfisis femoral distal, lo que puede ser un desafío particular para la planificación y el logro de la fijación del vástago. ${ }^{7}$ Se ha reconocido que los vástagos modulares no cementados pueden reconstruir el hueso femoral proximal con una alta tasa de consolidación, mientras que su complicación más frecuente es la inestabilidad..$^{35}$ Mulay y cols..$^{36}$ publicaron 10 FFP B2 y 14 B3 utilizando un vástago cónico, estriado, de fijación distal (Link MP Reconstruction Hip Stem, Waldemar Link GmbH \& Co, Hamburgo, Alemania). La tasa de consolidación de las fracturas fue del $91 \%$ y la tasa de luxación, del 21\%, no hubo casos de hundimiento. Del mismo modo, Moreta y cols. ${ }^{37}$ informaron 43 fracturas FFP (31 tipo B2 y 13 tipo B3), con una tasa de consolidación del 93\%, sin signos de aflojamiento a los cinco años de seguimiento; sin embargo, la inestabilidad fue la complicación más común (16,3\%), sin diferencias entre los tallos modulares y monobloque. A pesar de las múltiples opciones de versión, offset y longitud del vástago, la alta tasa de luxación todavía parece ser un problema en este grupo de pacientes añosos. Dos posibles causas serían el pinzamiento debido al exceso de tejido cicatricial del lado medial del fémur proximal ${ }^{36}$ y el hundimiento sutil del implante que genera un acortamiento y la pérdida de tensión de las partes blandas ${ }^{37}$.

Se ha recurrido a otras opciones para tratar este tipo de fracturas. Springer y cols. ${ }^{11}$ analizaron 118 revisiones femorales debidas a FFP y observaron que los vástagos cilíndricos totalmente recubiertos tenían una supervivencia superior que las prótesis parcialmente recubiertas y los vástagos cementados. Además, de las 18 cirugías de revisión por FFP con una aloprótesis o una prótesis tumoral, siete fracasaron debido a un aflojamiento aséptico y otra por una infección. ${ }^{11}$ En una de las series más grandes de FFP B3 tratadas con un aloinjerto femoral proximal ( 25 casos), Maury y cols. ${ }^{14}$ informaron resultados satisfactorios con un seguimiento medio de cinco años, aunque dos pacientes sufrieron una luxación y cuatro $(16 \%)$ requirieron una nueva cirugía de revisión. Dada su alta tasa de complicaciones, consideramos el uso de tales implantes cuando otras opciones terapéuticas hayan fallado.

En resumen, la reconstrucción femoral con la técnica de IOI tuvo una alta tasa de complicaciones posoperatorias para el tratamiento de las FFP Vancouver B3. Por este motivo, en nuestra práctica actual y debido a la exigente técnica quirúrgica, el IOI solo se indica en casos estrictamente seleccionados, sobre todo, en pacientes jóvenes con pérdida ósea masiva y una geometría del canal femoral desfavorable.

Conflicto de intereses: Los autores no declaran conflictos de intereses.

ORCID de J. I. Oñativia: https://orcid.org/0000-0001-8534-5101

ORCID de P. Slullitel: https://orcid.org/0000-0002-8957-075X

ORCID de M. Buttaro: https://orcid. org/0000-0003-3329-778X

\section{BIBLIOGRAFÍA}

1. Tsiridis E, Haddad FS, Gie GA. The management of periprosthetic femoral fractures around hip replacements. Injury 2003;34(2):95-105. https://doi.org/10.1016/s0020-1383(02)00257-7

2. Meek RMD, Norwood T, Smith R, Brenkel IJ, Howie CR. The risk of peri-prosthetic fracture after primary and revision total hip and knee replacement. J Bone Joint Surg Br 2011;93(1):96-101. https://doi.org/10.1302/0301-620X.93B1.25087

3. Kurtz S, Ong K, Lau E, Mowat F, Halpern M. Projections of primary and revision hip and knee arthroplasty in the United States from 2005 to 2030. J Bone Joint Surg Am 2007;89(4):780-5. https://doi.org/10.2106/JBJS.F.00222

4. Tsiridis E, Spence G, Gamie Z, El Masry MA, Giannoudis PV. Grafting for periprosthetic femoral fractures: strut, impaction or femoral replacement. Injury 2007;38(6):688-97. https://doi.org/10.1016/j.injury.2007.02.046

5. Brady OH, Garbuz DS, Masri BA, Duncan CP. The reliability and validity of the Vancouver classification of femoral fractures after hip replacement. J Arthroplasty 2000;15(1):59-62. https://doi.org/10.1016/s0883-5403(00)91181-1

6. Lindahl H, Malchau H, Herberts P, Garellick G. Periprosthetic femoral fractures classification and demographics of 1049 periprosthetic femoral fractures from the Swedish National Hip Arthroplasty Register. J Arthroplasty 2005;20(7):857-65. https://doi.org/10.1016/j.arth.2005.02.001 
7. Rodriguez JA, Berliner ZP, Williams CA, Robinson J, Hepinstall MS, Cooper HJ. Management of Vancouver type-B2 and B3 periprosthetic femoral fractures: restoring femoral length via preoperative planning and surgical execution using a cementless, tapered, fluted stem. JBJS Essent Surg Tech 2017;7(3):e27. https://doi.org/10.2106/JBJS.ST.17.00007

8. Garbuz DS, Toms A, Masri BA, Duncan CP. Improved outcome in femoral revision arthroplasty with tapered fluted modular titanium stems. Clin Orthop Relat Res 2006;453:199-202. https://doi.org/10.1097/01.blo.0000238875.86519.cf

9. Kwong LM, Miller AJ, Lubinus P. A modular distal fixation option for proximal bone loss in revision total hip arthroplasty: a 2- to 6-year follow-up study. J Arthroplasty 2003;18(3 Suppl 1):94-7. https://doi.org/10.1054/arth.2003.50083

10. Van Houwelingen AP, Duncan CP, Masri BA, Greidanus NV, Garbuz DS. High survival of modular tapered stems for proximal femoral bone defects at 5 to 10 years followup. Clin Orthop Relat Res 2013;471(2):454-62. https://doi.org/:10.1007/s11999-012-2552-8

11. Springer BD, Berry DJ, Lewallen DG. Treatment of periprosthetic femoral fractures following total hip arthroplasty with femoral component revision. J Bone Joint Surg Am 2003;85(11):2156-62. https://doi.org/10.2106/00004623-200311000-00015

12. Abdel MP, Cottino U, Mabry TM. Management of periprosthetic femoral fractures following total hip arthroplasty: a review. Int Orthop 2015;39(10):2005-10. https://doi.org/10.1007/s00264-015-2979-0

13. Khan T, Grindlay D, Ollivere BJ, Scammell BE, Manktelow ARJ, Pearson RG. A systematic review of Vancouver B2 and B3 periprosthetic femoral fractures. Bone Joint J 2017;99-B(4 Suppl B):17-25. https://doi.org/10.1302/0301-620X.99B4.BJJ-2016-1311.R1

14. Maury AC, Pressman A, Cayen B, Zalzal P, Backstein D, Gross A. Proximal femoral allograft treatment of Vancouver type-B3 periprosthetic femoral fractures after total hip arthroplasty. J Bone Joint Surg Am 2006;88(5):953-8. https://doi.org/10.2106/JBJS.E.00120

15. Berry DJ. Treatment of Vancouver B3 periprosthetic femur fractures with a fluted tapered stem. Clin Orthop Relat Res 2003;(417):224-31. https://doi.org/10.1097/01.blo.0000096821.67494.f6

16. Engelbrecht E, Heinert K. Klassifikation und Behandlungsrichtlinien von Knochensubsanzverlusten bei Revisionsoperationen am Huftgelenk mittelfrsige Ergebnisse. En: Primare und Revisionsalloarthroplastik HrsgEndo-Klinik, Hamburg. Berlin: Springer-Verlag; 1987:189-201.

17. Nuñez LV, Buttaro MA, Morandi A, Pusso R, Piccaluga F. Frozen sections of samples taken intraoperatively for diagnosis of infection in revision hip surgery. Acta Orthop 2007;78(2):226-30. https://doi.org/10.1080/17453670710013726

18. Mowe JC. Standards for tissue banking. Arlington, VA: American Association of Tissue Banks; 1988.

19. Gie GA, Linder L, Ling RS, Simon JP, Slooff TJ, Timperley AJ. Impacted cancellous allografts and cement for revision total hip arthroplasty. J Bone Joint Surg Br 1993;75(1):14-21. https://doi.org/10.1302/0301-620X.75B1.8421012

20. Salvati EA, Sharrock NE, Westrich G, Potter HG, Valle AGD, Sculco TP. The 2007 ABJS Nicolas Andry Award: three decades of clinical, basic, and applied research on thromboembolic disease after THA: rationale and clinical results of a multimodal prophylaxis protocol. Clin Orthop Relat Res 2007;459:246-54. https://doi.org/10.1097/BLO.0b013e31805b7681

21. Klein GR, Parvizi J, Rapuri V, Wolf CF, Hozack WJ, Sharkey PF, et al. Proximal femoral replacement for the treatment of periprosthetic fractures. J Bone Joint Surg Am 2005;87(8):1777-81. https://doi.org/10.2106/JBJS.D.02420

22. Park M-S, Lim Y-J, Chung W-C, Ham D-H, Lee S-H. Management of periprosthetic femur fractures treated with distal fixation using a modular femoral stem using an anterolateral approach. J Arthroplasty 2009;24(8):1270-6. https://doi.org/10.1016/j.arth.2009.07.013

23. Gruen TA, McNeice GM, Amstutz HC. "Modes of failure" of cemented stem-type femoral components: a radiographic analysis of loosening. Clin Orthop Relat Res 1979;(141):17-27. PMID: 477100

24. Loudon JR, Charnley J. Subsidence of the femoral prosthesis in total hip replacement in relation to the design of the stem. J Bone Joint Surg Br 1980;62(4):450-3. https://doi.org/10.1302/0301-620X.62B4.7430222

25. Engh CA, Massin P, Suthers KE. Roentgenographic assessment of the biologic fixation of porous-surfaced femoral components. Clin Orthop Relat Res 1990;(257):107-28. PMID: 2199114

26. Richards CJ, Garbuz DS, Masri BA, Duncan CP. Vancouver type B3 periprosthetic fractures: evaluation and treatment. Instr Course Lect 2009;58:177-81. PMID: 19385531 
27. Tsiridis E, Narvani AA, Haddad FS, Timperley JA, Gie GA. Impaction femoral allografting and cemented revision for periprosthetic femoral fractures. J Bone Joint Surg Br 2004;86(8):1124-32. https://doi.org/10.1302/0301-620x.86b8.14854

28. Li D, Hu Q, Kang P, Yang J, Zhou Z, Shen B, Pei F. Reconstructed the bone stock after femoral bone loss in Vancouver B3 periprosthetic femoral fractures using cortical strut allograft and impacted cancellous allograft. Int Orthop 2018 4;42(12):2787-95. https://doi.org/10.1007/s00264-018-3997-5

29. Wilson MJ, Hook S, Whitehouse SL, Timperley AJ, Gie GA. Femoral impaction bone grafting in revision hip arthroplasty: 705 cases from the originating centre. Bone Joint J 2016;98B(12):1611-9. https://doi.org/10.1302/0301-620X.98B12.37414

30. Ornstein E, Linder L, Ranstam J, Lewold S, Eisler T, Torper M. Femoral impaction bone grafting with the Exeter stem - the Swedish experience: survivorship analysis of 1305 revisions performed between 1989 and 2002. J Bone Joint Surg Br 2009;91(4):441-6. https://doi.org/10.1302/0301-620X.91B4.21319

31. Buttaro M, Comba F, Zanotti G, Piccaluga F. Fracture of the C-Stem cemented femoral component in revision hip surgery using bone impaction grafting technique: report of 9 cases. Hip Int 2015;25(2):184-7. https://doi.org/10.5301/hipint.5000210

32. Schreurs BW, Arts JJC, Verdonschot N, Buma P, Slooff TJJH, Gardeniers JWM. Femoral component revision with use of impaction bone-grafting and a cemented polished stem. Surgical technique. J Bone Joint Surg Am 2006;88 (Suppl 1)Pt 2:259-74. https://doi.org/10.2106/JBJS.F.00340

33. Te Stroet MAJ, Rijnen WHC, Gardeniers JWM, Schreurs BW, Hannink G. Predictors of unsuccessful outcome in cemented femoral revisions using bone impaction grafting; Cox regression analysis of 208 cases. Hip Int 2016;26(5):444-50. https://doi.org/10.5301/hipint.5000370

34. Hassaballa M, Mehendale S, Poniatowski S, Kalantzis G, Smith E, Learmonth ID. Subsidence of the stem after impaction bone grafting for revision hip replacement using irradiated bone. J Bone Joint Surg Br 2009;91(1):37-43. https://doi.org/10.1302/0301-620X.91B1.20376

35. Abdel MP, Lewallen DG, Berry DJ. Periprosthetic femur fractures treated with modular fluted, tapered stems. Clin Orthop Relat Res 2014;472(2):599-603. https://doi.org/10.1007/s11999-013-2936-4

36. Mulay S, Hassan T, Birtwistle S, Power R. Management of types B2 and B3 femoral periprosthetic fractures by a tapered, fluted, and distally fixed stem. J Arthroplasty 2005;20(6):751-6. https://doi.org/10.1016/j.arth.2004.11.020

37. Moreta J, Uriarte I, Ormaza A, Mosquera J, Iza K, Aguirre U, et al. Outcomes of Vancouver B2 and B3 periprosthetic femoral fractures after total hip arthroplasty in elderly patients. Hip Int 2019;29(2):184-90. https://doi.org/10.1177/1120700018772163 